\title{
Identification of biomarkers and construction of a microRNA-mRNA regulatory network for ependymoma using integrated bioinformatics analysis
}

\author{
BIAO YANG ${ }^{*}$, JUN-XI DAI", YUAN-BO PAN*, YAN-BIN MA and SHENG-HUA CHU \\ Department of Neurosurgery, Shanghai Ninth People's Hospital Affiliated to \\ Shanghai Jiao Tong University School of Medicine, Shanghai 201999, P.R. China
}

Received March 31, 2019; Accepted August 22, 2019

DOI: $10.3892 / 01.2019 .10941$

\begin{abstract}
Ependymomas (EPNs) are one of the most common types of malignant neuroepithelial tumors. In an effort to identify potential biomarkers involved in the pathogenesis of EPN, the mRNA expression profiles of the GSE25604, GSE50161, GSE66354, GSE74195 and GSE86574 datasets, in addition to the microRNA (miRNA/miR) expression profiles of GSE42657 were downloaded from the Gene Expression Omnibus (GEO) database. Differentially expressed genes (DEGs) and differentially expressed miRNAs (DEMs) between EPN and normal brain tissue samples were identified using the Limma package in $\mathrm{R}$ and GEO2R, respectively. Functional and pathway enrichment analyses were conducted using the Database for Annotation, Visualization and Integrated Discovery. A protein-protein interaction network was constructed using the Search Tool for Retrieval of Interacting Genes database, which was visualized using Cytoscape. The targeted genes of DEMs were predicted using miRWalk 2.0 and a miRNA-mRNA regulatory network was constructed. Following analysis, a total of 948 DEGs and 129 DEMs were identified. Functional
\end{abstract}

Correspondence to: $\mathrm{Dr}$ Sheng-Hua Chu, Department of Neurosurgery, Shanghai Ninth People's Hospital Affiliated to Shanghai Jiao Tong University School of Medicine, 280 Mohe Road, Baoshan, Shanghai 201999, P.R. China

E-mail: shenghuachu@126.com

*Contributed equally

Abbreviations: EPN, ependymoma; miRNA, microRNA; DEG, differentially expressed gene; GEO, Gene Expression Omnibus; DEM, differentially expressed miRNA; DAVID, Database for Annotation, Visualization and Integrated Discovery; PPI, protein-protein interaction; STRING, Search Tool for Retrieval of Interacting Genes; GO, Gene Ontology; KEGG, Kyoto Encyclopedia of Genes and Genomes; CC, cellular component; BP, biological process; $\mathrm{MF}$, molecular function; $\mathrm{CNS}$, central nervous system

Key words: ependymoma, differentially expressed gene, differentially expressed miRNA, module, regulatory network enrichment analysis revealed that 609 upregulated DEGs were significantly enriched in 'PI3K-Akt signaling pathway', while 339 downregulated DEGs were primarily involved in 'cell junction' and 'retrograde endocannabinoid signaling'. In addition, 6 hub genes [cyclin dependent kinase 1, CD44 molecule (Indian blood group) (CD44), proliferating cell nuclear antigen (PCNA), $M Y C$, synaptotagmin 1 (SYT1) and kinesin family member 4A] and 6 crucial miRNAs [homo sapiens (hsa)-miR-34a-5p, hsa-miR-449a, hsa-miR-106a-5p, $h s a-m i R-124-3 p$, hsa-miR-128-3p and hsa-miR-330-3p] were identified as biomarkers and potential therapeutic targets for EPN. Furthermore, a microRNA-mRNA regulatory network was constructed to highlight the interactions between DEMs and their target DEGs; this included the hsa-miR-449a-SYT1, hsa-miR-34a-5p-SYT1, hsa-miR-330-3p-CD44 and hsa-miR-124-3p-PCNA pairs, whose expression levels were confirmed using reverse transcription-quantitative polymerase chain reaction. In conclusion, the present study may provide important data for the investigation of the molecular mechanisms of EPN pathogenesis.

\section{Introduction}

Ependymomas (EPNs) are one of the most commonly diagnosed malignant neuroepithelial tumors in children and adults, accounting for $\sim 1.8 \%$ of all primary central nervous system (CNS) tumors (1). It has been demonstrated that ependymomas in children $<5$ years of age account for $>50 \%$ of all cases (2). Following comprehensive treatment with gross total resection and adjuvant radiotherapy, only $70 \%$ of patients with intracranial ependymoma $<5$ years of age were cured (3). In 2016, the latest World Health Organization classification for ependymoma was published as follows: Subependymoma; myxopapillary ependymoma; ependymoma; anaplastic ependymoma; and ependymoma, RELA proto-oncogene, NF- $\mathrm{B}$ subunit fusion-positive (4). However, the pathological criterion is limited for clinical application; it was demonstrated that $7 \%$ of cases were misdiagnosed and were subsequently reclassified as ependymoma, which is indicative of the requirement for a more precise molecular classification system (5). Therefore, an increased number of studies are required to improve our understanding of the molecular mechanisms involved in EPN. 
microRNAs (miRNAs/miR) are a class of non-protein-coding small single-stranded RNAs, which bind to the 3'-untranslated region (UTR) of target mRNAs, inhibiting gene expression at the post-transcriptional level $(6,7)$. It was suggested that high expression levels of $m i R-124-3 p$ significantly decreased the progression-free survival time of patients with EPN by negatively regulating tumor protein p53 nuclear protein 1 (TP53INP1), thereby indicating a potential role of $m i R-124-3 p$ as a therapeutic biomarker (8). Other studies involving microarray analyses revealed that the expression level of cyclin D1, which is involved in DNA repair, was increased in recurrent EPNs compared with the primary EPNs (9). With considerable advances in high-throughput technologies, increasing numbers of miRNAs and genes have been confirmed to serve important roles in the diagnosis, treatment and prognosis of EPNs, which may lead to further investigations into the molecular mechanisms of its pathogenesis.

In the present study, five gene expression profiles datasets (GSE25604, GSE50161, GSE66354, GSE74195 and GSE86574) and one miRNA expression profile dataset (GSE42657) were downloaded from the Gene Expression Omnibus (GEO) to identify differentially expressed genes (DEGs) and differentially expressed miRNAs (DEMs) between EPN samples and normal brain tissue samples. Then, Gene Ontology (GO) and Kyoto Encyclopedia of Genes and Genomes (KEGG) enrichment analyses, in addition to protein-protein interaction (PPI) network analyses of these DEGs, were performed to identify associated hub genes and modules. Furthermore, an miRNA-mRNA network analysis was conducted to identify the key miRNAs (and their targeted genes) that may be utilized as biomarkers of EPN. Taken together, the present study may provide an improved understanding of the genetic mechanisms of EPN.

\section{Materials and methods}

Microarray data. mRNA (GSE25604, GSE50161, GSE66354, GSE74195 and GSE86574) and miRNA (GSE42657) expression profiles were downloaded from the GEO database (http://www. ncbi.nlm.nih.gov/geo/); data from the GSE25604 dataset, including $15 \mathrm{EPN}$ and 7 normal samples, generated from GPL571 platform (Affymetrix Human Genome U133A 2.0 Array) were submitted on Nov 24, 2010 and updated on April 05, 2017. The GSE50161 (including 46 EPN and 13 normal samples), GSE66354 (including 64 EPN and 13 normal samples), GSE74195 (including 13 EPN and 5 normal samples) and GSE86574 (including 29 EPN and 10 normal samples) datasets were generated using the same microarray platform. miRNA expression data from the GSE42657 dataset were submitted on November 30, 2012 and updated on February 12, 2016. GSE42657, from the GPL8179 platform (illumina Human v2 MicroRNA expression beadchip) contained 14 EPN and 7 normal samples. Survival data of EPN from GEO and The Cancer Genome Atlas databases were not available.

Furthermore, 10 EPN and 10 normal brain tissue samples were obtained from the Department of Neurosurgery, Shanghai Ninth People's Hospital Affiliated to Shanghai Jiao Tong University School of Medicine (Shanghai, China). The present study was approved by the Ethics Committees of the Shanghai Ninth People's Hospital Affiliated to Shanghai Jiao Tong University School of Medicine, and informed patient consent was obtained prior to clinical research. All tissue samples were immediately frozen in liquid nitrogen and subsequently stored at $-80^{\circ} \mathrm{C}$.

Identification of DEGs and DEMs. Following transformation into gene symbols according to probe annotation files of each platform, the original expression data were preprocessed using Linear models for microarray data using Limma package in $\mathrm{R}$ (v.3.4.1; https://www.r-project.org/). This included background correction, quantile normalization, summarization and probe ID-to-gene symbol transformation.

GEO2R (http://www.ncbi.nlm.nih.gov/geo/geo2r/) is an online tool used to identify differentially expressed molecules among $\geq$ two sample groups in a GEO dataset. In the present study, GEO2R was used to identify DEGs and DEMs between EPN and normal samples, and a threshold of adjusted $\mathrm{P}<0.05$ and $\mid \log 2$ fold-change (FC) $\mid>1$ was applied. To control for Type I errors and false discovery rate, P-values were adjusted using the Benjamini \& Hochberg false discovery rate method provided by Limma package (10).

Functional and pathway enrichment analysis. Following data preprocessing and DEG acquisition, aberrantly expressed DEGs were uploaded to the Database for Annotation, Visualization and Integrated Discovery (DAVID; v.6.8; https://david.ncifcrf.gov/) for functional and pathway enrichment analysis (11). KEGG pathway database records networks of cellular molecular interactions, and variants of these interactions specific to particular organisms (12). GO enrichment analysis includes 'Biological process' (BP), 'Molecular function' (MF) and 'Cellular component' (CC). In addition, the criterion $\mathrm{P}<0.05$ was considered to indicate statistically significance. Reactome pathway analysis was also conducted (https://www.reactome.org/) (13), the results of which were visualized using Cytoscape 3.5.1 (14).

PPI network construction and module selection. Following uploading of the DEGs to the Search Tool for Retrieval of Interacting Genes (STRING) database (version 10.5; http://www.string-db.org/), a PPI network was constructed and visualized in Cytoscape (v.3.5.1) (14,15). Hub genes were identified using cytoHubba plug-in and DEG modules were distinguished using Molecular Complex Detection (MCODE) plug-in $(16,17)$. The criteria were set as scores $>3$ and nodes $>4$. Furthermore, in order to conduct GO Biological process (BP) and KEGG pathway enrichment analysis, DEGs in the selected modules were further analyzed using DAVID. Terms with $\mathrm{P}<0.05$ were considered to be significant. The transcription factor (TF)-gene regulatory network was performed using the iRegulion plug-in (18).

Identification of potential miRNA target genes. miRWalk2.0 (http://zmf.umm.uni-heidelberg.de/mirwalk2) comprises a collection of predicted and validated miRNA-target interactions. The predicted interactions were assessed using the following 12 miRNA target prediction programs: DIANA-microT v4.0 (19), DIANA-microT-CDS (20), miRanda-rel2010 (21), mirBridge (22), miRDB 4.0 (23), miRmap (24), miRNAMap (25), PicTar 2 (26), PITA (27), RNA22 v2 (28), RNAhybrid 2.1 (29) and Targetscan 6.2 (30). 
In order to improve prediction reliability, the genes predicted by $\geq 8$ of the databases among 12 databases were selected as the DEM targets. Validated databases were then used to search for verified targeted genes. Finally, the sums of predicted and validated genes, which were repeated with the obtained DEGs, were recognized as the eventual target genes of the identified DEMs.

Construction of a negative miRNA-mRNA regulatory network. miRNAs promote mRNA degradation or inhibit the translation of negatively regulated targeted genes (7). Therefore, a negative miRNA-mRNA regulatory network was selected and visualized in Cytoscape. The top 3 significant DEMs and their target hub genes were also identified.

Association analyses and efficacy evaluation. To verify the clinical value of the hub genes, association analyses and efficacy evaluation were conducting using mRNA (GSE25604, GSE50161, GSE66354, GSE74195 and GSE86574) and miRNA (GSE42657) expression profiles, respectively. The associations between hub genes and the corresponding clinical features were illustrated using by boxplots, and statistical significance was determined using an independent sample t-test. Efficacy evaluation was conducted by receiver operating characteristic (ROC) curve analysis with the 'pROC' package in R language. Genes with an area under the curve (AUC) value $>0.7$ were considered to distinguish tumor tissues from normal tissues.

Reverse transcription-quantitative $(R T-q) P C R$. Total RNA was extracted from tissues using TRIzol ${ }^{\circledR}$ reagent (Takara Biotechnology Co., Ltd.,) according to the manufacturer's protocol. RT-qPCR was performed using the FastStart essential DNA Green Master mix (Roche Diagnostics) and a miRNA qPCR Assay kit (CWBio Technology Co., Ltd.), and performed using the iCycler iQ Real-Time Detection System (Bio-Rad Laboratories, Inc.). snRNA U6 and GAPDH were used as internal controls for miR and mRNA, respectively. The expression levels were quantified using the $2^{-\Delta \Delta \mathrm{Cq}}$ method (31) and the fold change for target genes was normalized to the appropriate internal control. The primer sequences are listed in Table SI.

Statistical analysis. SPSS software (v.22.0; IBM Corp.), GraphPad Prism (v.7.0; GraphPad Software, Inc.) and R software (v.3.4.1) were used for statistical analysis. The statistical significance between EPN and normal brain tissues was determined using an independent sample t-test. $\mathrm{P}<0.05$ was considered to indicate a statistically significant difference. The bar plots were generated by GraphPad Prism or R software.

\section{Results}

Identification of DEGs. Following preprocessing, boxplots of the samples from five selected datasets are demonstrated in Fig. S1. Based on the cut-off criteria of $\mathrm{P}<0.05$ and $\mid \log 2 \mathrm{FCl}>1$, a total of 12,115, 6,030, 4,493, 3,026 and 7,751 DEGs between EPN and normal brain samples were identified from the GSE25604, GSE50161, GSE66354, GSE74195 and GSE86574 datasets, respectively (Fig. S2). Among these datasets, a total of 948 overlapping DEGs were identified (Fig. 1), including

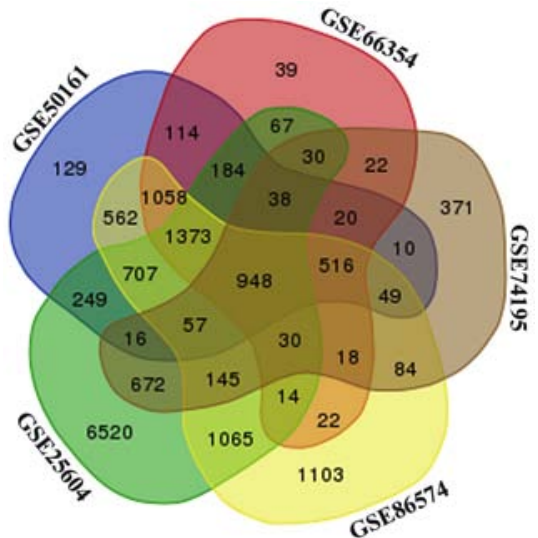

Figure 1. Venn diagram of differentially expressed genes among the five datasets.

609 upregulated and 339 downregulated genes, were screened out among the five datasets. In addition, the expression levels of 129 DEMs were identified as being significantly different between EPN and normal brain tissues, of which 39 were upregulated and 80 were downregulated miRNAs in EPN tissues (Table SII).

Functional and pathway enrichment analysis. Following uploading of the 609 upregulated and 339 downregulated DEGs to DAVID, GO categories and KEGG pathways with $\mathrm{P}<0.05$ were identified, and the top 5 significant terms were selected from corresponding GO categories on up- and downregulated DEGs (Table SIII). GO analysis results revealed that upregulated DEGs were significantly associated with 'extracellular exosome' and 'extracellular matrix' in CC, 'extracellular matrix organization' and 'cell adhesion' in BP, and 'extracellular matrix structural constituent' and 'protein binding' in MF. The downregulated DEGs were significantly enriched in 'cell junction' and 'plasma membrane' in $\mathrm{CC}$, 'chemical synaptic transmission' and 'neurotransmitter secretion' in $\mathrm{BP}$, and 'calcium ion binding' and 'GABA-A receptor activity' in MF.

In addition, the top 10 significantly enriched pathways of the up- and downregulated DEGs were selected from significant KEGG pathways (Table SIV). The upregulated DEGs were significantly enriched in 'ECM-receptor interaction', 'focal adhesion' and 'graft-versus-host disease', while downregulated DEGs were associated with 'GABAergic synapse', 'retrograde endocannabinoid signaling' and 'morphine addiction'. Reactome pathway analysis results indicated that DEGs were associated with 'G1/S-specific transcription' and 'NOTCH1 intracellular domain regulates transcription' (Fig. 2).

PPI network construction and module selection. A PPI network of 948 DEGs was produced using the STRING online tool, with a cutoff of score $>0.7$ (Fig. 3; Table SV). Using the cytoHubba plug-in, DEGs with the top 20 betweenness scores and with degrees $\geq 24$ (top 21) were identified as hub genes. A total of 6 nodes were selected as hub genes, including cyclin dependent kinase 1 (CDK1), CD44 molecule (Indian blood group) (CD44), proliferating cell nuclear antigen (PCNA), $M Y C$, synaptotagmin 1 (SYT1) and kinesin family member $4 \mathrm{~A}$ (KIF4A) (Table SVI). Among these 6 genes, betweenness 


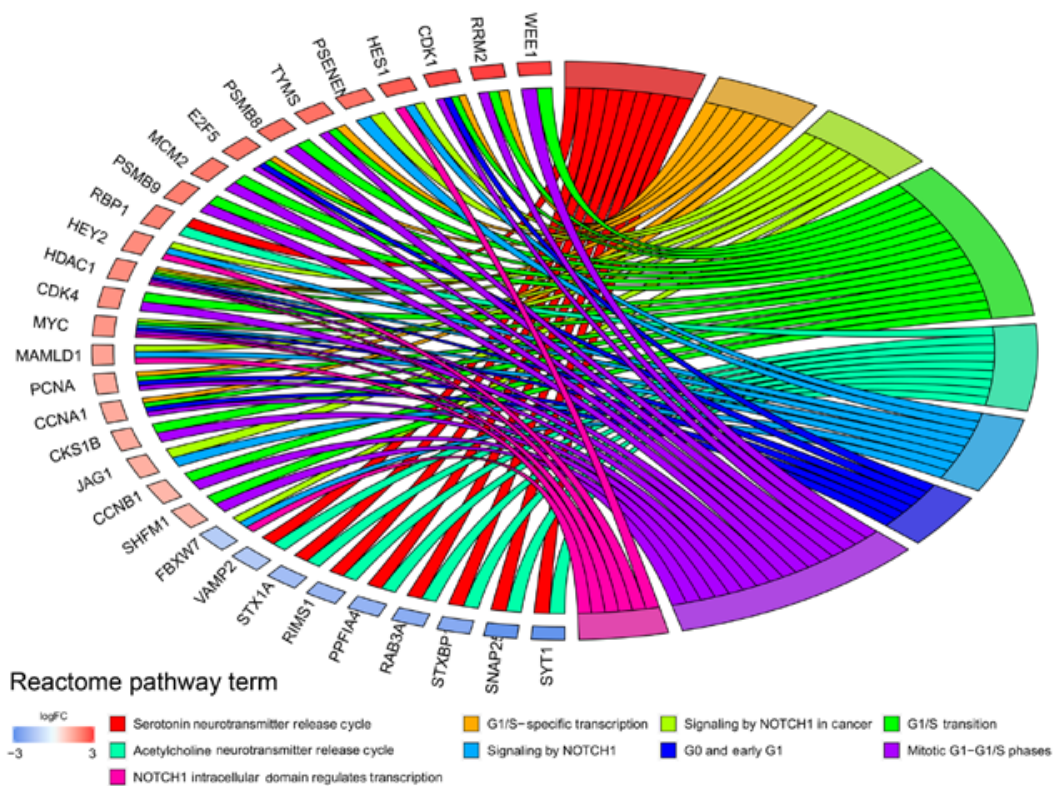

Figure 2. Significantly enriched Reactome pathway analysis of differentially expressed genes.

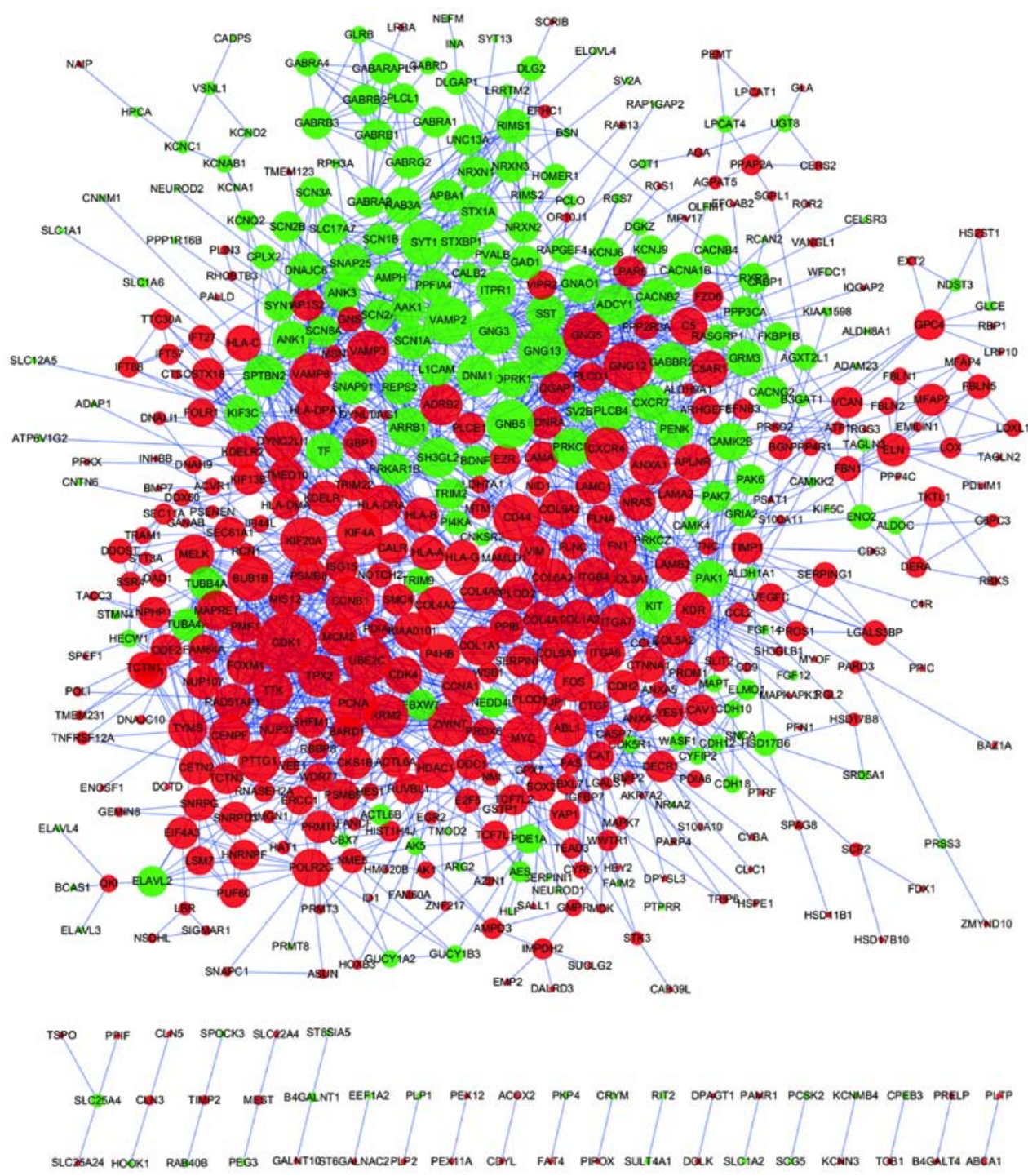

Figure 3. Protein-protein interaction regulatory network of DEGs in ependymoma. Nodes represent DEGs. Red nodes indicate upregulated DEGs and green nodes indicated downregulated DEGs. Nodes with higher degree values are depicted with larger shapes. Edges/lines stand for the regulatory association between any 2 nodes. DEGs, differentially expressed genes. 
Table I. miRNA-target pairs of top 3 miRNAs and hub genes from the miRNA-mRNA regulatory network.

\begin{tabular}{llll}
\hline Mature miRNA & \multicolumn{1}{c}{ Precursor miRNA } & Expression & Hub gene \\
\hline$h s a-m i R-449 a$ & $h s a-m i R-449 a$ & Up & SYT1 \\
$h s a-m i R-34 a-5 p$ & $h s a-m i R-34 a^{*} ; h s a-m i R-34 a$ & Up & SYT1 \\
$h s a-m i R-330-3 p$ & $h s a-m i R-330$ & Down & CD44 \\
$h s a-m i R-124-3 p$ & $h s a-m i R-124^{*}, h s a-m i R-124$ & Down & PCNA \\
\hline
\end{tabular}

miRNA, microRNA; hsa, homo sapiens; SYT1, synaptotagmin 1; CD44, CD44 molecule (Indian blood group); PCNA, proliferating cell nuclear antigen.

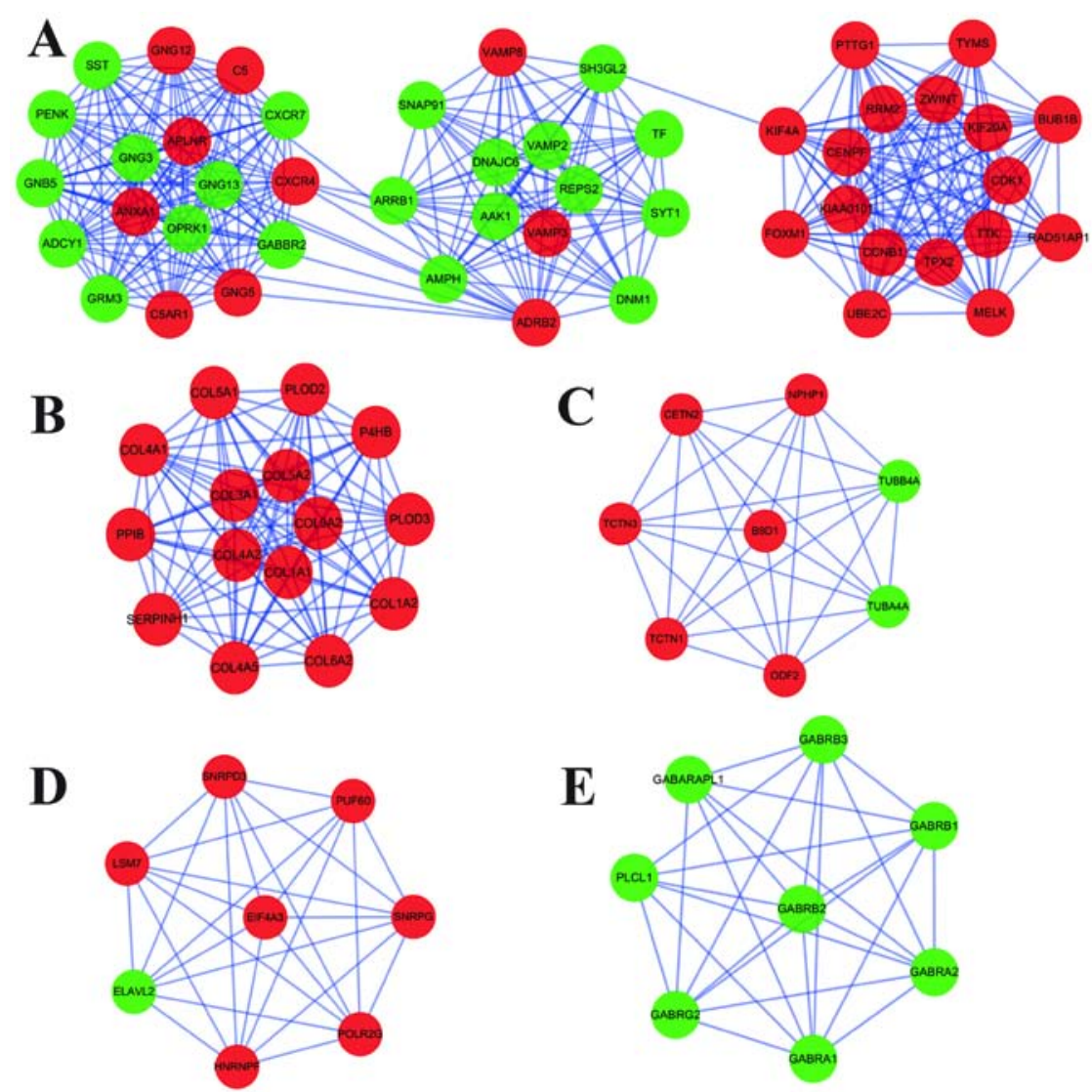

Figure 4. Top 5 modules in the protein-protein interaction network for DEGs. (A) Module 1. (B) Module 2. (C) Module 3. (D) Module 4. (E) Module 5. Nodes represent DEGs. Edges/lines stand for the regulation association between any 2 nodes. Red and green nodes represent upregulated and downregulated genes, respectively. DEGs, differentially expressed genes.

of $M Y C$ was the greatest, and at $48, C D K 1$ had the highest number of node degrees. The TF-gene regulatory network is demonstrated in Fig. S3.

Furthermore, MCODE analysis revealed a total of 19 available modules, from which the top 5 significant modules were selected (Fig. 4); BP and KEGG enrichment analysis of DEGs between these modules were subsequently performed (Table SVII). Enrichment analysis demonstrated that the DEGs in module 1 were primarily enriched in 'morphine addiction' and 'GABAergic synapse' (KEGG analysis), and 'chemical synaptic transmission' in the BP category of the GO analysis. In the KEGG pathway analysis, the DEGs in modules 2-5 were mostly associated with 'protein digestion and absorption', 'pathogenic Escherichia coli infection', 'spliceosome' and 'GABAergic synapse', respectively.
Construction of a negative miRNA-gene regulatory network. Among the 39 upregulated DEMs and 339 downregulated DEGs, 19,319 predicted and 16,116 validated miRNA-target pairs were identified. A total of 26,033 predicted and 15,986 validated miRNA-target pairs were also identified from 80 downregulated DEMs and 609 upregulated DEGs, respectively. Subsequently, 812 miRNA-DEG pairs from upregulated DEMs and downregulated DEGs were selected for the construction of a miRNA-DEG network, which was visualized using Cytoscape (Fig. 5). Similarly, a network including 1,232 miRNA-DEG pairs from downregulated DEMs and upregulated DEGs was also generated (Fig. 6).

Following analysis, upregulated homo sapiens (hsa)-miR-34a-5p, hsa-miR-449a and hsa-miR-106a-5p, were revealed to be the 3 most significantly 


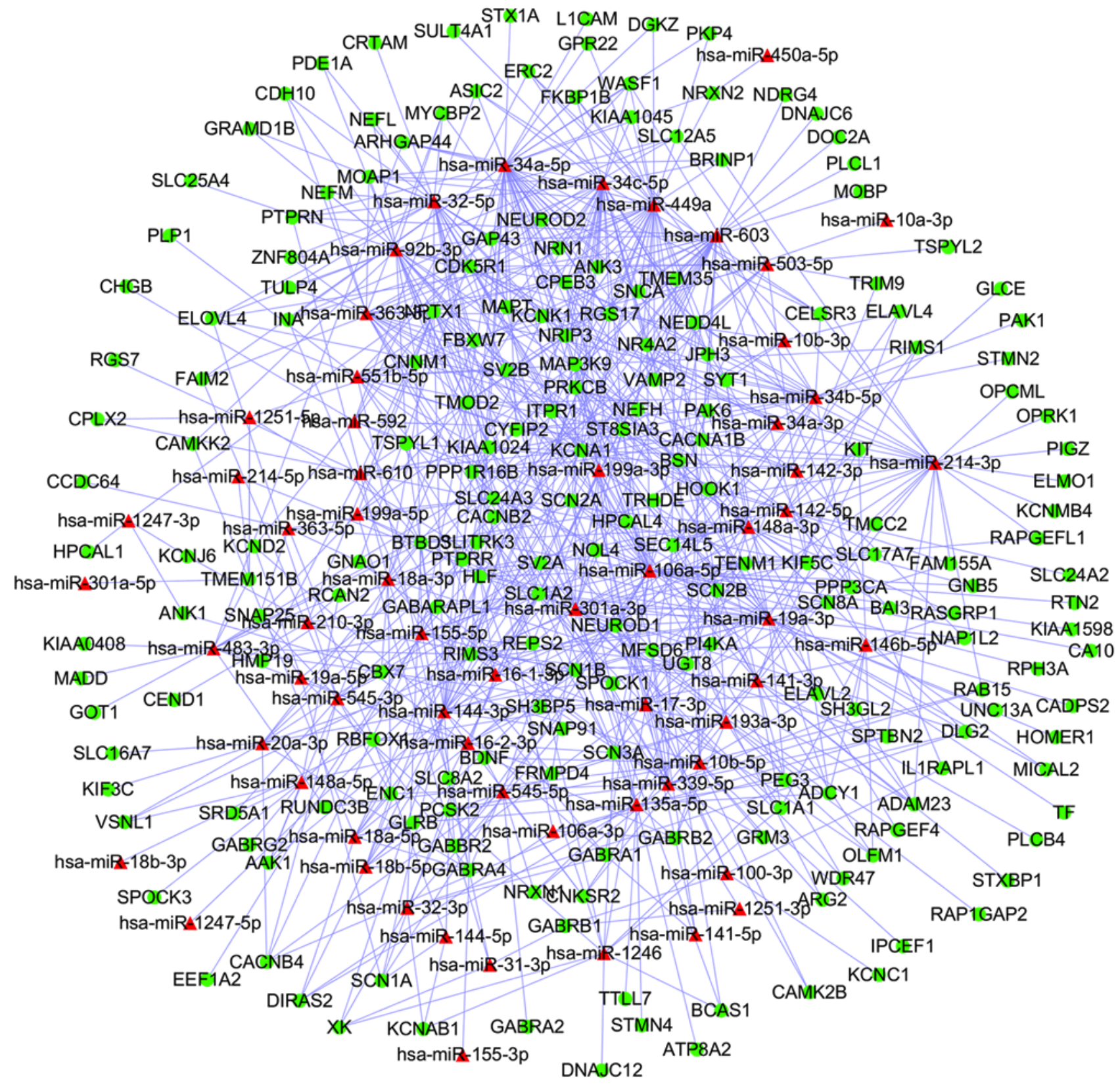

Figure 5. Regulatory network of upregulated miRNAs and downregulated DEGs in ependymoma. The red triangles represent the upregulated miRNAs. The green circles indicate downregulated DEGs. miRNA, microRNA; DEGs, differentially expressed genes.

upregulated miRs, whilst $h s a-m i R-124-3 p$, hsa-miR-128-3p and $h s a-m i R-330-3 p$ were identified as the top 3 downregulated miR. Finally, 4 miRNA-DEG pairs (hsa-miR-449a-SYT1, hsa-miR-34a-5p-SYT1, hsa-miR-330-3p-CD44 and hsa-miR-124-3p-PCNA) were identified (Table I).

Association analysis and evaluation of efficacy. Significant differences were observed in the expression levels of 3 mRNAs (SYT1, CD44 and PCNA) and 4 miRNAs (hsa-miR-449a, hsa-miR-34a-5p, hsa-miR-330-3p and hsa-miR-124-3p) between EPN and normal tissues $(\mathrm{P}<0.05$; (Fig. 7). The independent sample t-test and AUC analyses confirmed that each of mRNA and miRNA (including hsa-miR-449a, hsa-miR-34a-5p, hsa-miR-330-3p and hsa-miR-124-3p) possessed high specificity and sensitivity values, indicating their potentials as prognostic biomarkers (Fig. 8).
RT-qPCR analysis. Compared with the normal tissue samples, the expression levels of CD44, PCNA, hsa-miR-124-3p and hsa-miR-330-3p were significantly upregulated $(\mathrm{P}<0.01)$, and those of SYT1, hsa-miR-34a-5p and hsa-miR-449a were significantly downregulated $(\mathrm{P}<0.01)$ in EPN tissues. This was consistent with the results of the integrated bioinformatics analyses (Fig. 9).

\section{Discussion}

In previous years, microarray and next-generation sequencing technologies have developed rapidly, promoting more in-depth investigation into the molecular mechanisms of EPN. Evidence to indicate that miRNAs negatively regulate mRNA expression, and subsequently affect the development and progression of tumors, has been described (6,7). In the present study, 948 


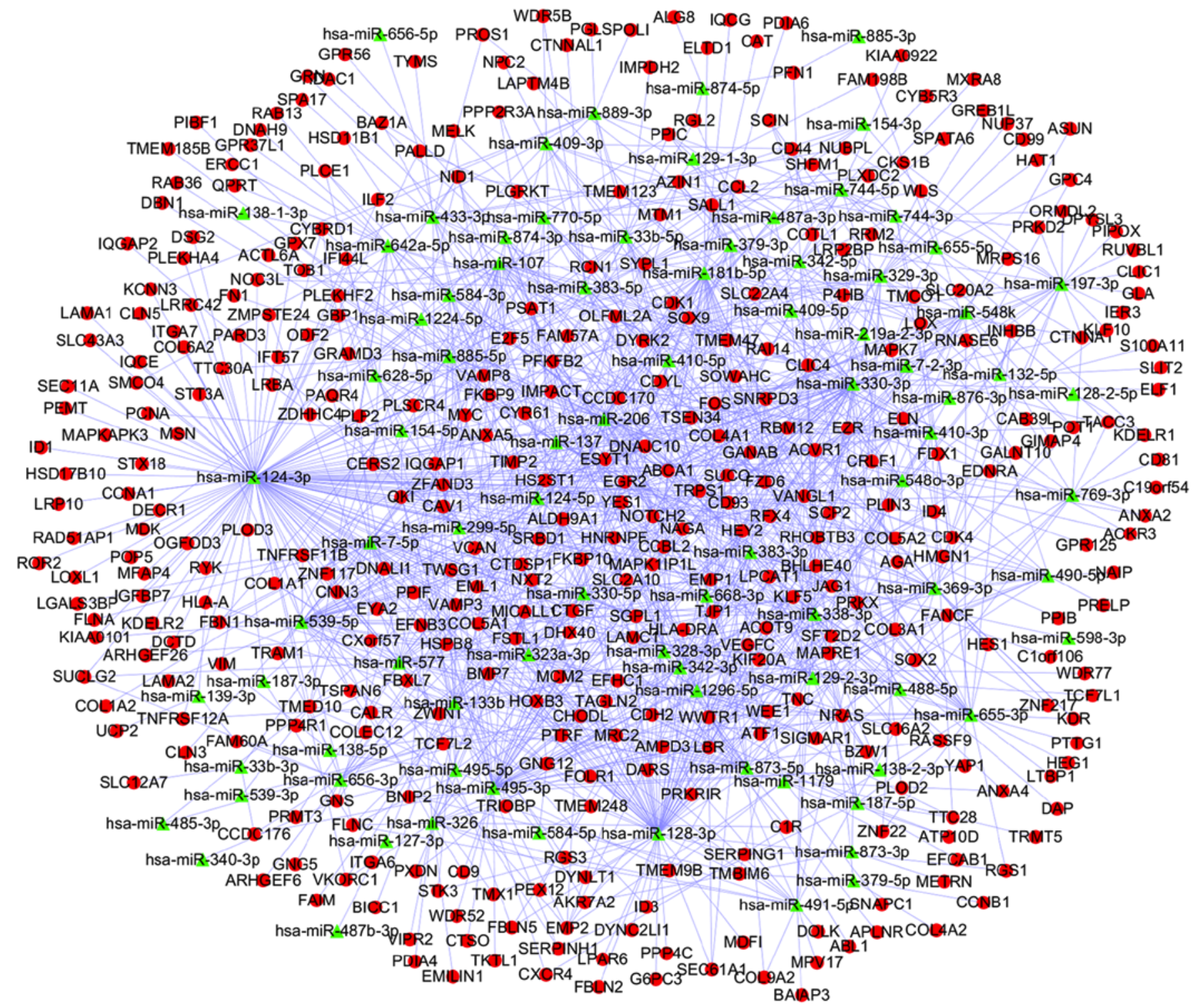

Figure 6. Regulatory network between downregulated miRNAs and upregulated DEGs in ependymoma. The green triangles represent downregulated miRNAs. The red circles indicate upregulated DEGs. miRNA, microRNA; DEGs, differentially expressed genes.

DEGs and 129 DEMs were selected from the GEO in an attempt to identify the molecular mechanisms and potential biomarkers of EPN.

GO and KEGG analysis results provided the functional and pathway information for 948 DEGs and the top 5 modules identified. These genes were primarily associated with 'cell division', 'mitotic nuclear division' and 'G2/M transition of mitotic cell cycle', which implied a regulatory function in mitosis. Additionally, 'retrograde endocannabinoid signaling', 'morphine addiction' and 'nicotine addiction', identified using KEGG pathway analysis, indicated that EPN may be significantly associated with cannabinoids, morphine and nicotine. The 'response to drug' term, obtained from the GO analysis, was associated with 28 upregulated DEGs, which indicated a potential drug resistance mechanism and provided potential targets for patients with EPN exhibiting chemotherapeutic tolerance. The PI3K-Akt signaling pathway was has been suggested to be important in glioblastoma and medulloblastoma, which supports the results of the present study $(32,33)$.
Furthermore, following the integrated analysis, 6 hub genes (including CDK1, CD44, PCNA, MYC, SYT1 and KIF4A) were identified from the PPI network of DEGs. PCNA is an immunohistochemical marker of cellular proliferation in tumors, and is therefore able to predict the survival outcome of patients with EPN (34). The myc oncogenes comprise 3 principal genes: $\mathrm{C}-m y c, \mathrm{~N}-m y c$ and L-myc. Fluorescence in situ hybridization and immunohistochemistry data have indicated that C-myc, a targeted gene of the Notch pathway, was significantly correlated with the development of adult onset EPNs (35). Also, increased C-myc expression has been associated with poor prognosis in patients with low-grade tumors (36). Although not previously confirmed, an increasing number of studies have demonstrated the importance of the 4 hub genes (CDK1, CD44, SYT1 and KIF4A) in diseases, particularly CNS tumors (37-41). CDK1, a member of the cyclin-dependent kinase $(C D K)$ family, serves an important role in G2-M phase transition, which is consistent with the GO analysis results of the present study (37). Following cDNA array and immunohistochemical analyses, a significant 
A

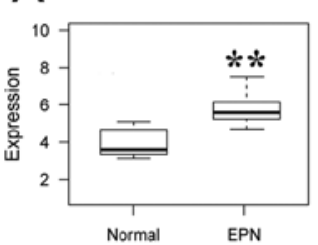

GSE25604

B

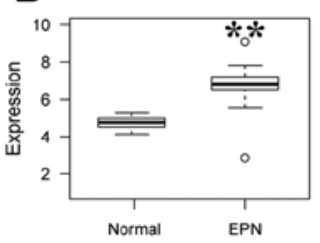

GSE25604

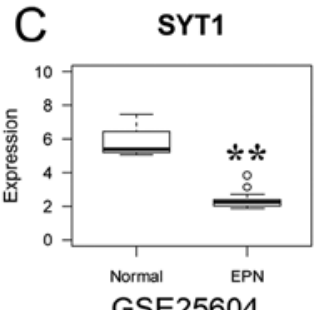

GSE25604
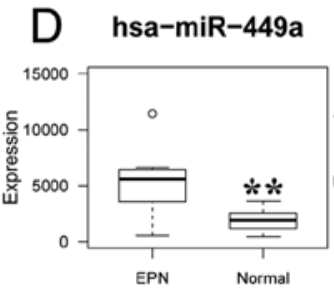

GSE42657
CD44

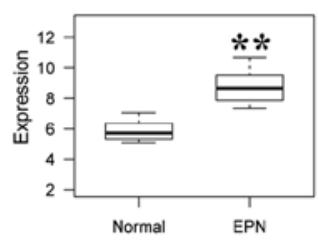

GSE50161

PCNA

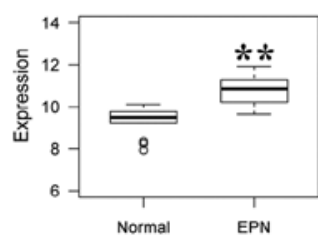

GSE50161

SYT1
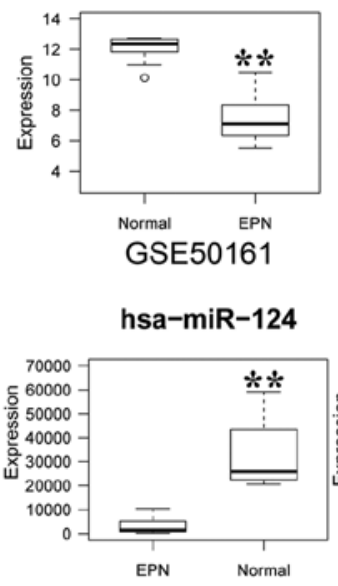

GSE42657
CD44
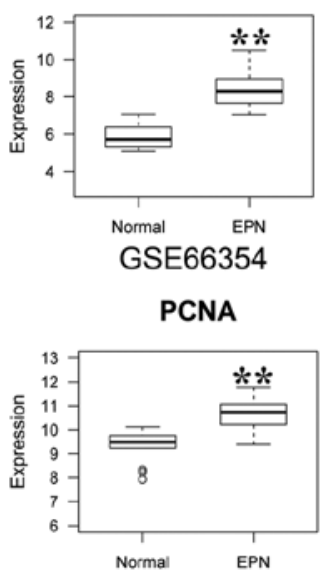

GSE66354

SYT1

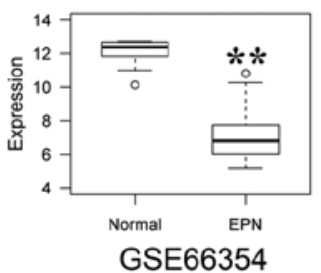

GSE66354
CD44
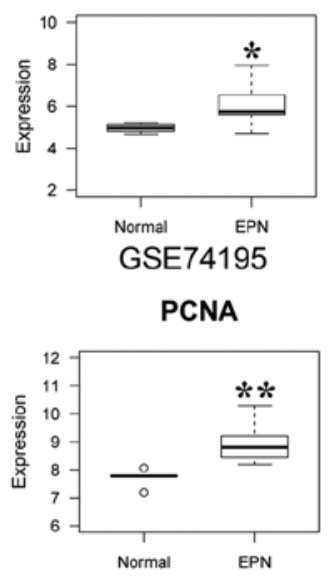

GSE74195

SYT1

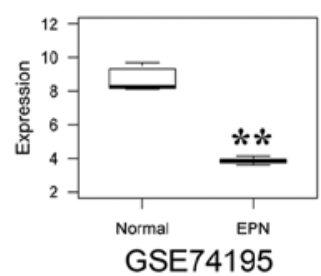

hsa-miR-330-3p

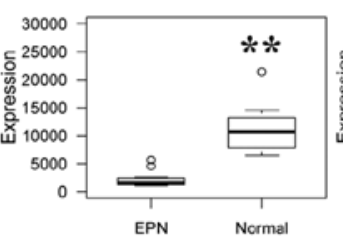

GSE42657

hsa-miR-34a-5p

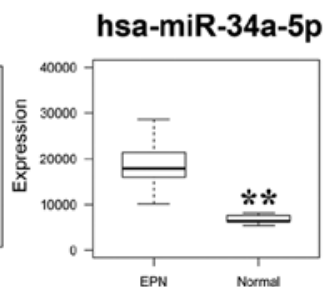

GSE42657

Figure 7. Boxplots of association analyses. Boxplots of (A) SYT1, (B) CD44 and (C) PCNA in the GSE25604, GSE50161, GSE66354, GSE74195 and GSE86574 datasets. (D) Boxplots of hsa-miR-449a, hsa-miR-34a-5p, hsa-miR-330-3p and hsa-miR-124-3p from the GSE42657. "P<0.05 and ${ }^{* *} \mathrm{P}<0.01$. SYT1, synaptotagmin 1; CD44, CD44 molecule (Indian blood group); PCNA, proliferating cell nuclear antigen; hsa, homo sapiens; miR, microRNA.

positive correlation was revealed between the expression level of $C D K 1$ and glioma oncogenesis (38). CD44 was also suggested to be a confirmed biomarker for distinguishing the molecular subtype of glioblastoma multiforme (39). In conclusion, the present study identified 6 hub genes that serve essential roles in EPN, and which may function as notable diagnostic and therapeutic biomarkers.

miRNAs are able to bind to the 3'-UTRs of specific genes to inhibit translation or promote the degradation of the corresponding mRNAs (7). Increasing evidence has indicated that miRNA dysregulation is responsible for the pathogenesis of EPN. In the present study, 6 miRNAs and 4 miRNA-DEG pairs were identified. Previous data has demonstrated that the expression level of $m i R-34 a-5 p$ was downregulated in colorectal cancer, which repressed apoptosis and cell cycle arrest at the G1 phase, and promoted p53 transcription to suppress tumor recurrence (42). $C D 117$ was also identified as a direct target of miR-34-5p in the progression of osteosarcoma (43). Additionally, the overexpression of miR-449a in glioblastoma inhibited myc-associated zinc-finger protein activity through the PI3K/AKT pathway, which highlighted a novel miRNA biomarker (44). Zhi et al $(45,46)$ revealed that miR-106a-5p served as a tumor suppressor in astrocytoma by inhibiting Fas-activated serine/threonine kinase, and that its expression was negatively correlated with clinical outcome. miR-124-3p was identified as a potential therapeutic and prognostic biomarker of EPN, which is consistent with the results of the present study, and further inhibited its target, TP53INPI, affecting clinical outcome (8). A study has indicated that $m i R-128-3 p$ overexpression promoted neuronal survival in ischemia-induced brain injury (47). Additionally, miR-128-3p was demonstrated to be a suppressive biomarker in various malignancies, including lung cancer, acute lymphoblastic leukemia and hepatocellular carcinoma (48-50). miR-330-3p knockdown was also discovered to inhibit tumor growth, in contrast to the effect produced by its overexpression (51). Notably, Pantaleo et al (52) identified 3 miRNA-mRNA regulatory networks, including the $m i R-330-3 p-C D 44$ pair, as biomarkers for the treatment of tyrosine protein kinase $\mathrm{KIT} /$ platelet derived growth factor receptor $\alpha$ wild type succinate dehydrogenase-deficient gastrointestinal stromal tumors (GISTs), which supported the results of the present study. However, the complex regulatory mechanisms of miRNAs in EPN remains to be fully elucidated. 

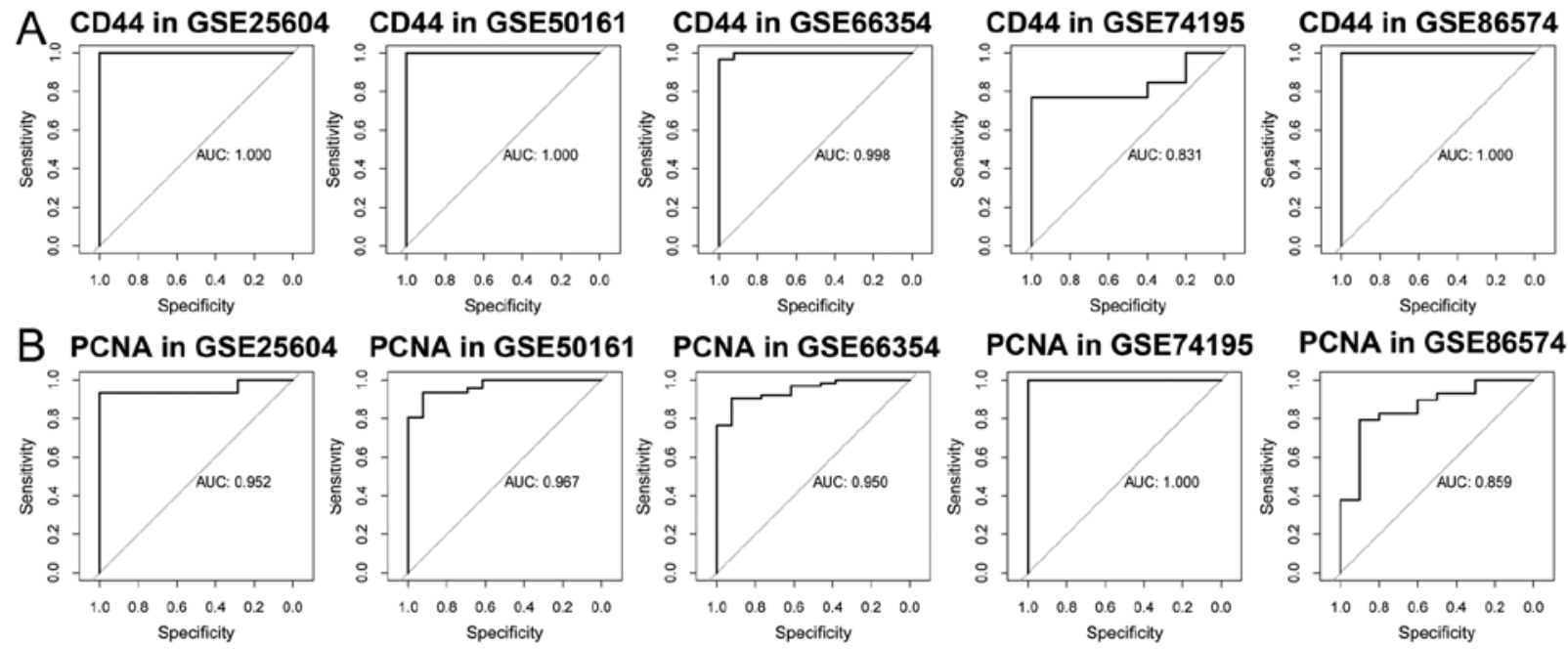

PCNA in GSE74195
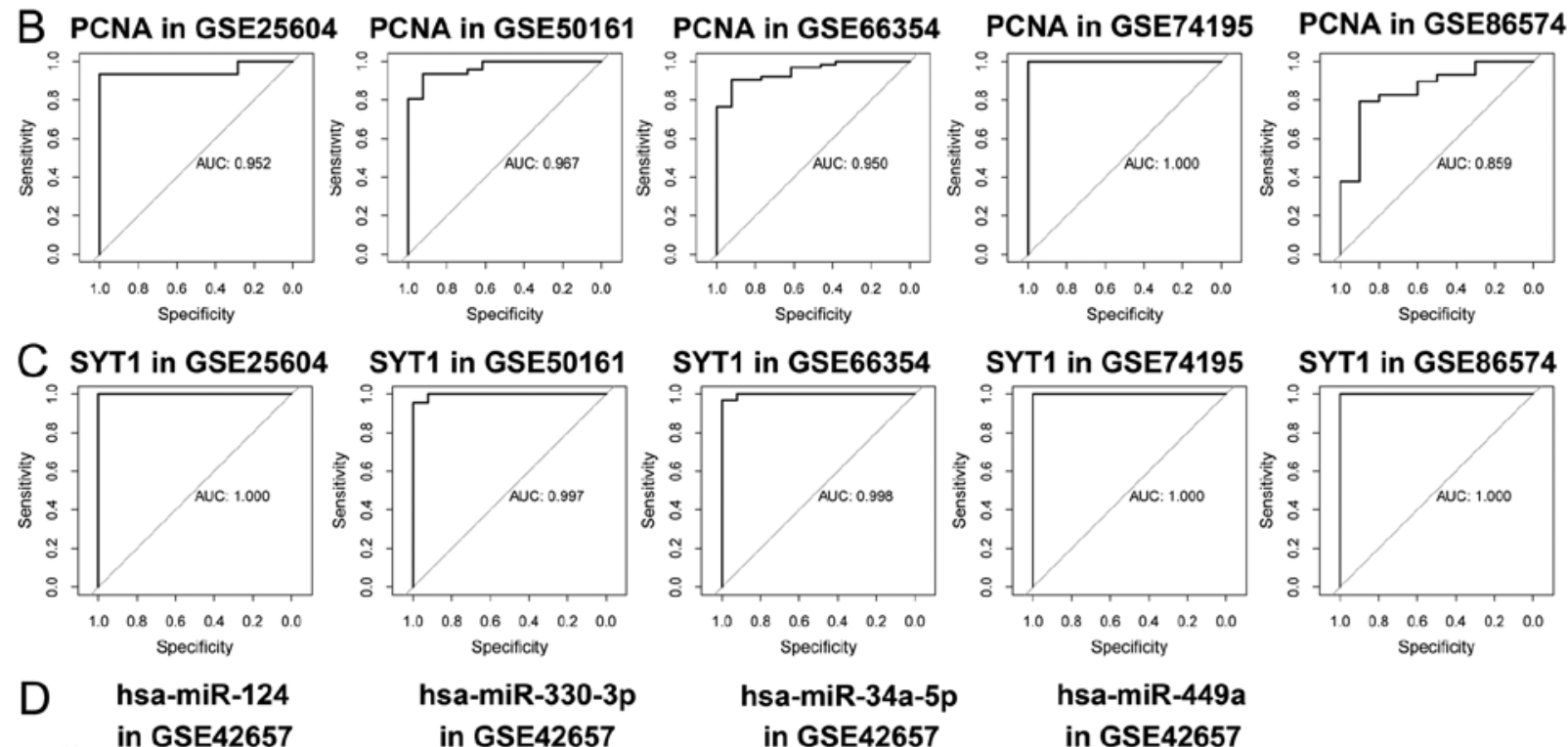

hsa-miR-330-3p in GSE42657
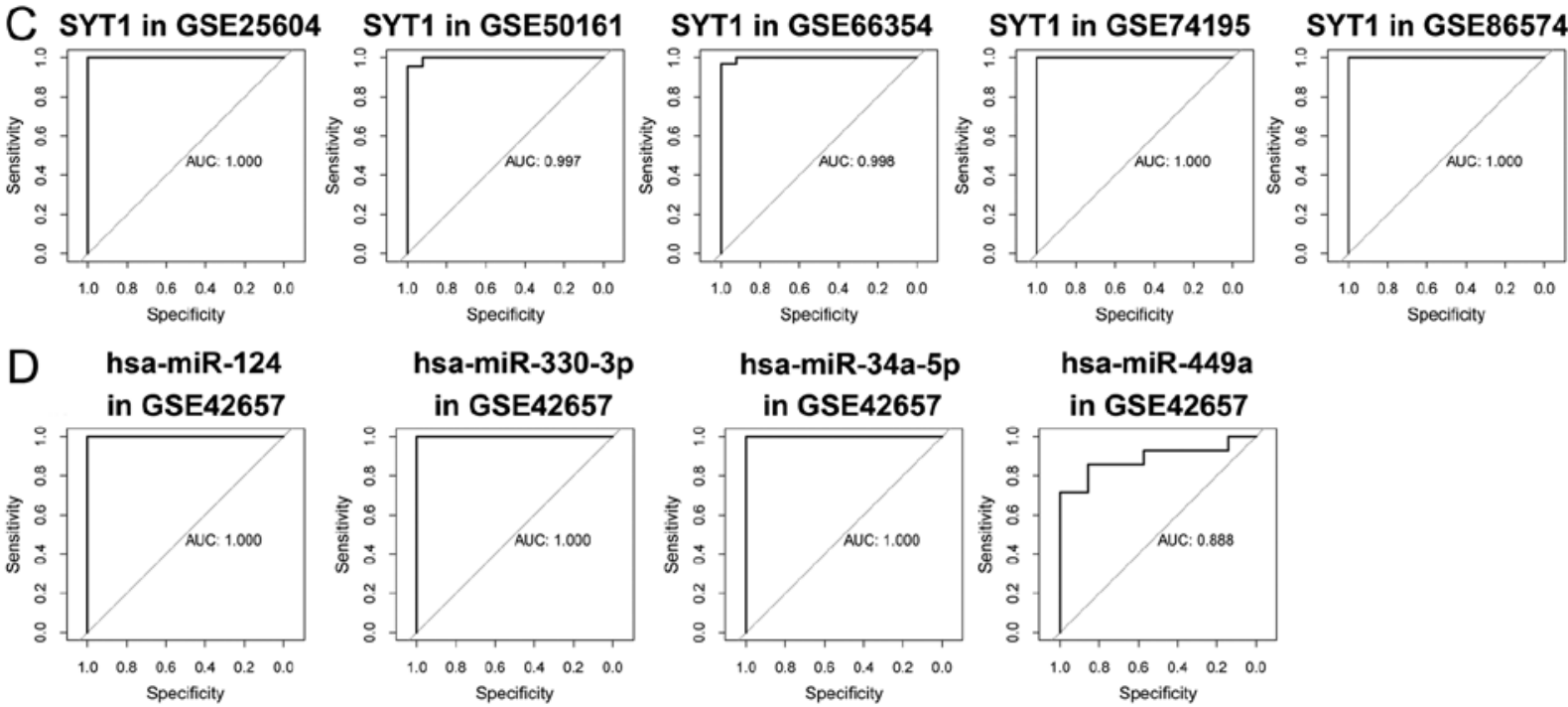

hsa-miR-449a in GSE42657

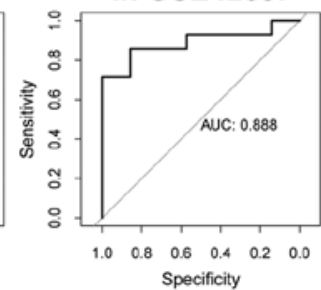

Figure 8. ROC analyses for efficacy evaluation. ROC analyses of (A) SYT1, (B) CD44 and (C) PCNA in the GSE25604, GSE50161, GSE66354, GSE74195 and GSE86574 datasets. (D) ROC analyses of hsa-miR-449a, hsa-miR-34a-5p, hsa-miR-330-3p and hsa-miR-124-3p from the GSE42657. AUC, area under the curve; hsa-miR, homo sapiens microRNA; ROC, receiver operating characteristic; SYT1, synaptotagmin 1; CD44, CD44 molecule (Indian blood group); PCNA, proliferating cell nuclear antigen; hsa, homo sapiens; miR, microRNA.
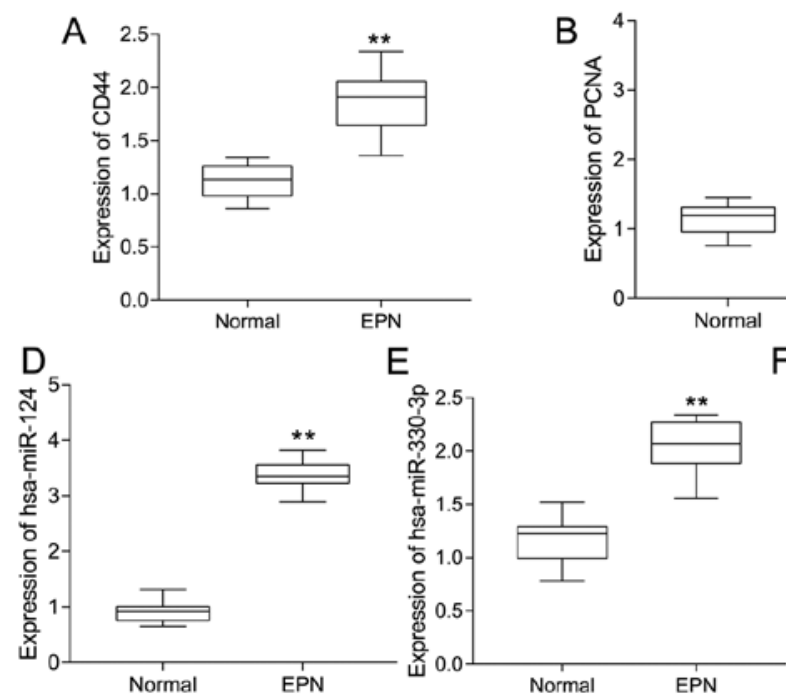

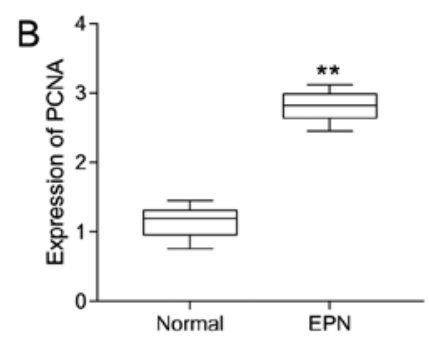

$\mathrm{F}$

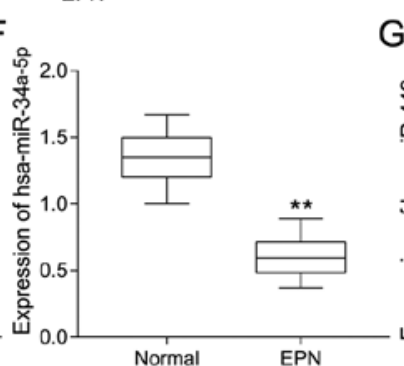

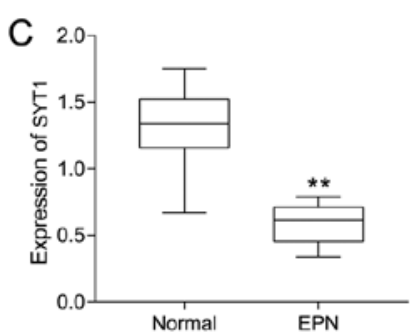

G

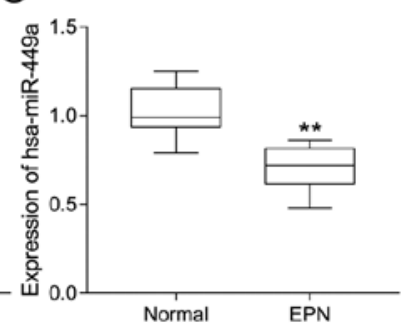

Figure 9. Boxplots of hub genes and miRs analyzed using reverse transcription-quantitative polymerase chain reaction. Significance between normal ( $\mathrm{n}=10$ ) and EPN tissues (n=10) for genes and miRs, including (A) CD44, (B) PCNA, (C) SYT1, (D) hsa-miR-124-3p, (E) hsa-miR-330-3p, (F) hsa-miR-34a-5p and (G) hsa-miR-449a, was determined using an independent sample t-test. ${ }^{* *} \mathrm{P}<0.01$. miRs, microRNAs; hsa, homo sapiens; EPN, ependymoma; CD44, CD44 molecule (Indian blood group); PCNA, proliferating cell nuclear antigen; SYT1, synaptotagmin 1. 
In conclusion, using integrated bioinformatics analysis, the present study revealed 948 DEGs and 129 DEMs associated with EPN. The results indicated 6 hub genes, 5 main modules, 6 crucial miRNAs and 4 miRNA-DEG pairs as novel potential biomarkers, which may facilitate further understanding of the molecular mechanisms of EPN. However, the functional value of these conclusions in EPN requires additional study.

\section{Acknowledgements}

Not applicable.

\section{Funding}

The present study was supported by the Natural Science Foundation of Shanghai (grant no. 19ZR1429800), Shanghai Jiao Tong University Medicine-Engineering Cross Research Foundation (grant no. YG2015MS25) and the Research Foundation of Shanghai No. 3 People's Hospital Affiliated to Shanghai Jiao Tong University School of Medicine (grant no. syz2015-015).

\section{Availability of data and materials}

The datasets generated and/or analyzed during the current study are available the GEO database repository (http://www. ncbi.nlm.nih.gov/geo/).

\section{Authors' contributions}

SHC and BY designed the study. JXD, YBP and YBM performed the statistical analyses and interpreted the data. BY and YBM wrote the manuscript. All authors read and approved the final manuscript.

\section{Ethics approval and consent to participate}

The present study was approved by the ethics committee of the Shanghai Ninth People's Hospital Affiliated to Shanghai Jiao Tong University School of Medicine (Shanghai, China).

\section{Patient consent for publication}

Informed patient consent was obtained prior to the commencement of clinical research.

\section{Competing interests}

The authors declare that they have no competing interests.

\section{References}

1. Ostrom QT, Gittleman H, de Blank PM, Finlay JL, Gurney JG, McKean-Cowdin R, Stearns DS, Wolff JE, Liu M, Wolinsky Y, et al: American brain tumor association adolescent and young adult primary brain and central nervous system tumors diagnosed in the united states in 2008-2012. Neuro Oncol 18 (Suppl 1): i1-i50, 2016.

2. Kilday JP, Rahman R, Dyer S, Ridley L, Lowe J, Coyle B and Grundy R: Pediatric ependymoma: Biological perspectives. Mol Cancer Res 7: 765-786, 2009.
3. Li AM,Dunham C, Tabori U, Carret AS, McNeely PD, Johnston D, Lafay-Cousin L, Wilson B, Eisenstat DD, Jabado N, et al: EZH2 expression is a prognostic factor in childhood intracranial ependymoma: A Canadian Pediatric Brain Tumor Consortium study. Cancer 121: 1499-1507, 2015.

4. Louis DN, Perry A, Reifenberger G, von Deimling A, Figarella-Branger D, Cavenee WK, Ohgaki H, Wiestler OD, Kleihues P and Ellison DW: The 2016 world health organization classification of tumors of the central nervous system: A summary. Acta neuropathol 131: 803-820, 2016.

5. Vera-Bolanos E, Aldape K, Yuan Y, Wu J, Wani K, Necesito-Reyes MJ, Colman H, Dhall G, Lieberman FS, Metellus $\mathrm{P}$, et al: Clinical course and progression-free survival of adult intracranial and spinal ependymoma patients. Neuro Oncol 17: 440-447, 2015.

6. Zhang B, Pan X and Anderson TA: MicroRNA: A new player in stem cells. J Cell Physiol 209: 266-269, 2006.

7. Fabian MR, Sonenberg N and Filipowicz W: Regulation of mRNA translation and stability by microRNAs. Annu Rev Biochem 79: 351-379, 2010.

8. Margolin-Miller Y, Yanichkin N, Shichrur K, Toledano H, Ohali A, Tzaridis T, Michowitz S, Fichman-Horn S, Feinmesser M, Pfister SM, et al: Prognostic relevance of miR-124-3p and its target TP53INP1 in pediatric ependymoma. Genes Chromosomes Cancer 56: 639-650, 2017.

9. Liang ML, Hsieh TH, Liu YR, Chen YW, Lee YY, Chang FC, Lin SC, Huang MC, Donald Ming-Tak H, Wong TT, et al: Significance of cyclin D1 overexpression in progression and radio-resistance of pediatric ependymomas. Oncotarget 9: 2527-2542, 2018.

10. Benjamini Y and Hochberg Y: Controlling the false discovery rate: A practical and powerful approach to multiple testing. J Royal Statistical Soc 57: 289-300, 1995.

11. Huang da W, Sherman BT and Lempicki RA: Bioinformatics enrichment tools: Paths toward the comprehensive functional analysis of large gene lists. Nucleic Acids Res 37: 1-13, 2009.

12. Kanehisa M: The KEGG database. Novartis Found Symp 247: 91-103, 119-128, 244-152, 2002.

13. Fabregat A, Sidiropoulos K, Garapati P, Gillespie M, Hausmann K, Haw R, Jassal B, Jupe S, Korninger F, McKay S, et al: The Reactome pathway Knowledgebase. Nucleic Acids Res 44: D481-487, 2016.

14. Shannon P, Markiel A, Ozier O, Baliga NS, Wang JT, Ramage D, Amin N, Schwikowski B and Ideker T: Cytoscape: A software environment for integrated models of biomolecular interaction networks. Genome Res 13: 2498-2504, 2003.

15. Szklarczyk D, Franceschini A, Kuhn M, Simonovic M, Roth A, Minguez P, Doerks T, Stark M, Muller J, Bork P, et al: The STRING database in 2011: Functional interaction networks of proteins, globally integrated and scored. Nucleic Acids Res 39: D561-D568, 2011.

16. Bader GD and Hogue CW: An automated method for finding molecular complexes in large protein interaction networks. BMC Bioinformatics 4: 2, 2003.

17. Chin $\mathrm{CH}$, Chen SH, Wu HH, Ho CW, Ko MT and Lin CY: cytoHubba: Identifying hub objects and sub-networks from complex interactome. BMC Syst Biol 8 (Suppl 4): S11, 2014

18. Janky R, Verfaillie A, Imrichova H, Van de Sande B, Standaert L, Christiaens V, Hulselmans G, Herten K, Naval Sanchez M, Potier D, et al: iRegulon: From a gene list to a gene regulatory network using large motif and track collections. PLoS Comput Biol 10: e1003731, 2014.

19. Maragkakis M, Vergoulis T, Alexiou P, Reczko M, Plomaritou K, Gousis M, Kourtis K, Koziris N, Dalamagas T and Hatzigeorgiou AG: Hatzigeorgiou DIANA-microT Web server upgrade supports Fly and Worm miRNA target prediction and bibliographic miRNA to disease association. Nucleic Acids Res 39: W145-W158, 2011.

20. Paraskevopoulou MD, Georgakilas G, Kostoulas N, Vlachos IS, Vergoulis T, Reczko M, Filippidis C, Dalamagas $T$ and Hatzigeorgiou AG: DIANA-microT web server v5.0: Service integration into miRNA functional analysis workflows. Nucleic Acids Res 41: W169-W173, 2013.

21. Betel D, Wilson M, Gabow A, Marks DS and Sander C: The microRNA.org resource: Targets and expression. Nucleic Acids Res 36: D149-D53, 2008.

22. Tsang JS, Ebert MS and van Oudenaarden A: Genome-wide dissection of microRNA functions and cotargeting networks using gene set signatures. Mol Cell 38: 140-153, 2010.

23. Wong N and Wang X: miRDB: An online resource for microRNA target prediction and functional annotations. Nucleic Acids Res 43: D146-D152, 2015 
24. Vejnar CE, Blum $M$ and Zdobnov EM: miRmap web: Comprehensive microRNA target prediction online. Nucleic Acids Res 41: W165-W168, 2013.

25. Hsu PW, Huang HD, Hsu SD, Lin LZ, Tsou AP, Tseng CP, Stadler PF, Washietl S and Hofacker IL: miRNAMap: Genomic maps of microRNA genes and their target genes in mammalian genomes. Nucleic Acids Res 34: D135-D139, 2006.

26. Krek A, Grün D, Poy MN, Wolf R, Rosenberg L, Epstein EJ, MacMenamin P, da Piedade I, Gunsalus KC, Stoffel M and Rajewsky N: Combinatorial microRNA target predictions. Nat Genet 37: 495-500, 2005.

27. Kertesz M, Iovino N, Unnerstall U, Gaul U and Segal E: The role of site accessibility in microRNA target recognition. Nat Genet 39: 1278-1284, 2007.

28. Miranda KC, Huynh T, Tay Y, Ang YS, Tam WL, Thomson AM, Lim B and Rigoutsos I: A pattern-based method for the identification of MicroRNA binding sites and their corresponding heteroduplexes. Cell 126: 1203-1217, 2006.

29. Krüger J and Rehmsmeier M: RNAhybrid: microRNA target prediction easy, fast and flexible. Nucleic Acids Res 34: W451-W454, 2006.

30. Garcia DM, Baek D, Shin C, Bell GW, Grimson A and Bartel DP: Weak seed-pairing stability and high target-site abundance decrease the proficiency of lsy- 6 and other microRNAs. Nat Struct Mol Biol 18: 1139-1146, 2011.

31. Livak KJ and Schmittgen TD: Analysis of relative gene expression data using real-time quantitative PCR and the 2(-Delta Delta C(T)) method. Methods 25: 402-408, 2001.

32. Li X, Wu C, Chen N, Gu H, Yen A, Cao L, Wang E and Wang L: $\mathrm{PI} 3 \mathrm{~K} / \mathrm{Akt} / \mathrm{mTOR}$ signaling pathway and targeted therapy for glioblastoma. Oncotarget 7: 33440-33450, 2016.

33. Dimitrova V and Arcaro A: Targeting the PI3K/AKT/mTOR signaling pathway in medulloblastoma. Curr Mol Med 15: 82-93, 2015.

34. Verstegen MJ, Leenstra DT, Ijlst-Keizers H and Bosch DA: Proliferation- and apoptosis-related proteins in intracranial ependymomas: An immunohistochemical analysis. J Neurooncol 56: 21-28, 2002.

35. Gupta RK, Sharma MC, Suri V, Kakkar A, Singh M and Sarkar C: Study of chromosome 9q gain, Notch pathway regulators and Tenascin-C in ependymomas. J Neurooncol 116: 267-274, 2014.

36. Faria C, Miguens J, Antunes JL, Salgado D, Nunes S, Barroso C, Martins Mdo C, Nunes VM and Roque L: Pediatric brain tumors: Genetics and clinical outcome. J Neurosurg Pediatr 5: 263-270, 2010.

37. Chen X, Zhang FH, Chen QE, Wang YY, Wang YL, He JC and Zhou J: The clinical significance of CDK1 expression in oral squamous cell carcinoma. Med Oral Patol Oral Cir Bucal 20: e 7-12, 2015.

38. Chen H, Huang Q, Zhai DZ, Dong J, Wang AD and Lan Q: CDK1 expression and effects of CDK1 silencing on the malignant phenotype of glioma cells. Zhonghua Zhong Liu Za Zhi 29 484-488, 2007 (In Chinese).

39. Brown DV, Daniel PM, D'Abaco GM, Gogos A, Ng W, Morokoff AP and Mantamadiotis T: Coexpression analysis of CD133 and CD44 identifies proneural and mesenchymal subtypes of glioblastoma multiforme. Oncotarget 6: 6267-6280, 2015.
40. Yang J, Hou Z, Wang C, Wang $\mathrm{H}$ and Zhang $\mathrm{H}$ : Gene expression profiles reveal key genes for early diagnosis and treatment of adamantinomatous craniopharyngioma. Cancer Gene Ther 25: 227-239, 2018.

41. Zhong S, Wu B, Dong X, Han Y, Jiang S, Zhang Y, Bai Y, Luo SX, Chen Y, Zhang $\mathrm{H}$ and Zhao G: Identification of driver genes and key pathways of glioblastoma shows JNJ-7706621 as a novel antiglioblastoma drug. World Neurosurg 109: e329-e342, 2018.

42. Gao J, Li N, Dong Y, Li S, Xu L, Li X, Li Y, Li Z, Ng SS, Sung JJ, et al: miR-34a-5p suppresses colorectal cancer metastasis and predicts recurrence in patients with stage II/III colorectal cancer. Oncogene 34: 4142-4152, 2015.

43. Pu Y, Zhao F, Wang H, Cai W, Gao J, Li Y and Cai S: MiR-34a-5p promotes the multi-drug resistance of osteosarcoma by targeting the CD117 gene. Oncotarget 7: 28420-28434, 2016.

44. Yao Y, Ma J, Xue Y, Wang P, Li Z, Li Z, Hu Y, Shang X and Liu Y: MiR-449a exerts tumor-suppressive functions in human glioblastoma by targeting Myc-associated zinc-finger protein. Mol Oncol 9: 640-656, 2015.

45. Zhi F, Zhou G, Shao N, Xia X, Shi Y, Wang Q, Zhang Y, Wang R, Xue L, Wang S, et al: miR-106a-5p inhibits the proliferation and migration of astrocytoma cells and promotes apoptosis by targeting FASTK. PLoS One 8: e72390, 2013.

46. Zhi F, Chen X, Wang S, Xia X, Shi Y, Guan W, Shao N, Qu H, Yang C, Zhang Y, et al: The use of hsa-miR-21, hsa-miR-181b and hsa-miR-106a as prognostic indicators of astrocytoma. Eur J Cancer 46: 1640-1649, 2010.

47. Mao G, Ren P, Wang G, Yan F and Zhang Y: MicroRNA-128-3p protects mouse against cerebral ischemia through reducing $\mathrm{p} 38 \mathrm{a}$ mitogen-activated protein kinase activity. J Mol Neurosci 61: 152-158, 2017.

48. Zhang R, Liu C, Niu Y, Jing Y, Zhang H, Wang J, Yang J, Zen K, Zhang J, Zhang CY and Li D: MicroRNA-128-3p regulates mitomycin C-induced DNA damage response in lung cancer cells through repressing SPTAN1. Oncotarget 8: 58098-58107, 2017.

49. Mets E, Van Peer G, Van der Meulen J, Boice M, Taghon T, Goossens S, Mestdagh P, Benoit Y, De Moerloose B, Van Roy N, et al: MicroRNA-128-3p is a novel oncomiR targeting PHF6 in T-cell acute lymphoblastic leukemia. Haematologica 99: 1326-1333, 2014

50. Huang CY, Huang XP, Zhu JY, Chen ZG, Li XJ, Zhang XH, Huang S, He JB, Lian F, Zhao YN, et al: miR-128-3p suppresses hepatocellular carcinoma proliferation by regulating PIK3R1 and is correlated with the prognosis of HCC patients. Oncol Rep 33: 2889-2898, 2015.

51. Meng H, Wang K, Chen X, Guan X, Hu L, Xiong G, Li J and Bai Y: MicroRNA-330-3p functions as an oncogene in human esophageal cancer by targeting programmed cell death 4 . Am J Cancer Res 5: 1062-1075,2015.

52. Pantaleo MA, Ravegnini G, Astolfi A, Simeon V, Nannini M, Saponara M, Urbini M, Gatto L, Indio V, Sammarini G, et al: Integrating miRNA and gene expression profiling analysis revealed regulatory networks in gastrointestinal stromal tumors. Epigenomics 8: 1347-1366, 2016.

This work is licensed under a Creative Commons Attribution-NonCommercial-NoDerivatives 4.0 International (CC BY-NC-ND 4.0) License. 